\title{
Myostatin-deficient mice exhibit reduced insulin resistance through activating the AMP-activated protein kinase signalling pathway
}

\author{
C. Zhang $\cdot$ C. McFarlane $\cdot$ S. Lokireddy $\cdot$ S. Bonala $~$ \\ X. Ge • S. Masuda • P. D. Gluckman • M. Sharma • \\ R. Kambadur
}

Received: 21 September 2010 / Accepted: 18 January 2011 / Published online: 24 February 2011

(C) Springer-Verlag 2011

\begin{abstract}
Aims/hypothesis Myostatin-null mice $\left(\mathrm{Mstn}^{-1-}\right)$ have reduced body fat and increased tolerance to glucose. To date the molecular mechanisms through which myostatin regulates body fat content and insulin sensitivity are not known. Therefore, the aim of the current study was to identify signalling pathways through which myostatin regulates insulin sensitivity.

Methods Wild-type (WT) mice and $\mathrm{Mstn}^{-1-}$ mice were fed either a control chow diet or a high fat diet (HFD) for 12 weeks. Glucose tolerance testing and insulin stimulated glucose uptake by $\mathrm{M}$. extensor digitorum longus (EDL) were used as variables to determine insulin sensitivity. Quantitative PCR, Western blotting and enzyme assays
\end{abstract}

C. Zhang and C. McFarlane contributed equally to this study.

Electronic supplementary material The online version of this article (doi:10.1007/s00125-011-2079-7) contains supplementary material, which is available to authorised users.

C. Zhang • C. McFarlane · S. Masuda • P. D. Gluckman •

R. Kambadur

Growth, Development and Metabolism Program,

Singapore Institute for Clinical Sciences,

Singapore, Republic of Singapore

C. Zhang $\cdot \mathrm{S}$. Lokireddy $\cdot \mathrm{S}$. Bonala $\cdot \mathrm{X} . \mathrm{Ge} \cdot \mathrm{R}$. Kambadur $(\triangle)$

Department of Genomics and Genetics,

School of Biological Sciences, Nanyang Technological University,

60 Nanyang Drive,

637551, Singapore, Republic of Singapore

e-mail: KRavi@ntu.edu.sg

\section{Sharma}

Department of Biochemistry, YLL School of Medicine,

National University of Singapore,

Singapore, Republic of Singapore were used to monitor AMP-activated protein kinase (AMPK) levels and activity.

Results $\mathrm{Mstn}^{-/}$mice exhibited reduced fat accumulation and peripheral insulin resistance when compared with WT mice, even when they were fed an HFD. Furthermore, treatment with a myostatin antagonist also increased insulin sensitivity during HFD. Consistent with increased insulin sensitivity, we also detected elevated levels of GLUT4, AKT, p-AKT and insulin receptor substrate-1 in $\mathrm{Mstn}^{-/}$ muscles. Molecular analysis showed that there is increased expression and activity of AMPK in $\mathrm{Msth}^{-/-}$muscles. Furthermore, we also observed an increase in the AMPK downstream target genes, Sirt1 and Pgc-1 $\alpha$ (also known as Ppargcla), in skeletal muscle of $\mathrm{Mstn}^{-/}$mice.

Conclusions/interpretation We conclude that myostatin inactivation leads to increased AMPK levels and activity resulting in increased insulin sensitivity of skeletal muscle. We propose that, by regulating AMPK in skeletal muscle and adipose tissues, myostatin plays a major role in regulating insulin signalling.

Keywords p-AKT - AMPK - Glucose - GLUT4 - Insulin • Myostatin · PGC-1 $\alpha \cdot$ PRKAB1 $\cdot$ SLC2A $4 \cdot$ SIRT1

$\begin{array}{ll}\text { Abbreviations } \\ \text { ACC } & \text { Acetyl-CoA carboxylase } \\ \text { ActIIBR } & \text { Activin type IIB receptor } \\ \text { sActIIBR } & \text { Soluble activin type IIB receptor } \\ \text { AMPK } & \text { AMP-activated protein kinase } \\ \text { CD } & \text { Chow diet } \\ \text { CPT-1 } & \text { Carnitine palmitoyl transferase-1 } \\ \text { EDL } & \text { M. extensor digitorum longus } \\ \text { GTT } & \text { Glucose tolerance test } \\ \text { HFD } & \text { High fat diet }\end{array}$




\begin{tabular}{|c|c|}
\hline IACUC & Institutional Animal Care and Use Committ \\
\hline ITT & Insulin tolerance test \\
\hline MSTN & Myostatin \\
\hline qPCR & Quantitative real-time PCR \\
\hline PGC- $1 \alpha$ & $\begin{array}{l}\text { Peroxisome proliferator-activated receptor- } \\
\text { gamma coactivator- } 1 \text { alpha }\end{array}$ \\
\hline SAMS & SNF1/AMPK substrate \\
\hline SIRT1 & Sirtuin1 \\
\hline SLC2A4 & $\begin{array}{l}\text { Solute carrier family } 2 \text { (facilitated glucose } \\
\text { transporter) }\end{array}$ \\
\hline TBST & Tris buffered saline Tween 20 \\
\hline WAT & White adipose tissue \\
\hline $\mathrm{T}$ & Wild-type \\
\hline
\end{tabular}

\section{Introduction}

Myostatin (MSTN) is a growth and differentiating factor that belongs to the TGF- $\beta$ superfamily. While a high level of Mstn gene expression is detected in skeletal muscle [1], a low level of Mstn expression is also detected in other tissues including adipose tissue [1] and heart [2]. Inactivation of the Mstn gene in mice [1] or mutations in the bovine [3, 4], ovine [5] and human [6] Mstn gene result in a similar phenotype of increased muscle growth. Therefore, it is believed that MSTN is a negative regulator of skeletal myogenesis and its function is highly conserved during evolution. Functionally, MSTN not only regulates the proliferation and differentiation phases of myoblast growth $[7,8]$, but also controls the activation and further proliferation of muscle stem cells, the satellite cells [9]. MSTN has been shown to bind to the activin type IIB receptor (ActIIBR) and, therefore, either overproduction of a dominant negative form of ActIIBR in muscle [10] or treatment of mice with a soluble form of ActIIBR [11] (sActIIBR) have been shown to result in increased musculature.

The physiological effects of Mstn deletion are not restricted to skeletal muscle. A number of studies have reported significant decreases in the amount of adipose tissue in association with loss of MSTN function [12-14]. A flurry of recent research also suggests that loss of, or reduction in, MSTN levels leads to increased insulin sensitivity. In fact, suppression of MSTN function in mice, through transgenic overproduction of the MSTN propeptide, results in resistance to high fat dietary-induced obesity and insulin resistance [15]. This finding is further supported by studies on insulin resistant human participants, which show increased levels of MSTN in muscle and plasma [16]. Interestingly, short-term administration of recombinant MSTN protein has also been shown to induce insulin resistance in mice [16]. In extremely obese human participants it was further discovered that there were elevated levels of MSTN protein in both muscle and plasma samples [17]. In addition, injection of MSTNspecific antibodies along with exercise results in significantly increased insulin sensitivity [18]. Recently, Guo et al. showed that inhibition of MSTN signalling, specifically in adipose tissue, had no effect on glucose and insulin tolerance [12]. Contrary to this, muscle-specific inactivation of MSTN resulted in increased lean mass and improved glucose metabolism on both standard and high fat diets (HFDs) [12]. Although it is clear that lack of MSTN affects glucose uptake and metabolism, the specific mechanisms and signalling events in skeletal muscle and adipose tissue that help overcome insulin resistance in $\mathrm{Msth}^{-/-}$mice are not clearly understood.

Recently, a series of investigations has delineated the role of AMPK (also known as PRKAB1) in insulin sensitivity and resistance. AMPK is a heterotrimeric serine/threonine kinase, which consists of a catalytic $\alpha$ subunit and regulatory $\beta$ - and $\gamma$-subunits [19-21]. In skeletal muscle, $\alpha 2[22,23]$ is the major catalytic isoform produced, which forms a heterotrimeric complex with $\beta 2$ and $\gamma 3$ subunits. AMPK acts as a metabolic switch, which becomes activated by acute increases in the cellular AMP/ ATP ratio, such as occurs following exercise, or during conditions such as hypoxia, ischaemia or osmotic stress [24]. When intracellular ATP levels are low, AMPK switches off ATP-consuming processes, such as glycogen, fatty acid, and protein synthesis pathways, and activates alternative pathways for ATP regeneration, which include glucose transport, glycolysis and fatty acid oxidation. Activation of AMPK has also been shown to improve insulin sensitivity resulting in enhanced insulin-mediated glucose uptake in skeletal muscle [25-27].

In the current manuscript we have identified the molecular mechanisms that are responsible for the increased insulin sensitivity in $\mathrm{Msth}^{-/-}$mice. Here for the first time we show that there is increased abundance of AMPK in skeletal muscle, adipose tissue and liver, leading to an overall increase in glucose uptake and reduced fat accumulation in $\mathrm{Mstn}^{-/-}$mice, despite being fed on an HFD. This in turn leads to the increased sensitivity to insulin in $\mathrm{Mstn}^{--}$mice. We further show that treatment with an MSTN antagonist also reduces insulin resistance in mice fed on an HFD.

\section{Methods}

Animals Wild-type (WT) C57BL/6 mice were obtained from the Centre for Animal Resources (National University 
of Singapore, Singapore). $\mathrm{Mstn}^{-1-}$ mice (C57BL/6 background) were gifted from Se-Jin Lee (Johns Hopkins University, Baltimore, MD). The Institutional Animal Care and Use Committee (IACUC), Singapore, approved all experiments involving animals. Seven-week-old male $\mathrm{Msth}^{-1-}$ mice and WT littermates were housed in groups and maintained on standard chow diet (CD) at a constant temperature $\left(20^{\circ} \mathrm{C}\right)$ under a $12 \mathrm{~h} / 12 \mathrm{~h}$ artificial light/dark cycle with unlimited access to water until commencement of the trial. Two groups, consisting of five WT and five $\mathrm{Mstn}^{-/-}$mice each, were either fed HFD (45\% energy from fat, $21.3 \%$ energy from protein, $23.6 \%$ energy from fat and $41.2 \%$ energy from carbohydrates [58V8, TestDiet, Richmond, IN, USA]) or CD (10\% energy from fat; $17.3 \%$ energy from protein, $4.3 \%$ energy from fat and $67.4 \%$ energy from carbohydrates [58Y2, TestDiet]) for 12 weeks. For MSTN antagonist studies, 7-week-old male WT mice were fed the same CD or HFD (see above) for a period of 12 weeks together with injection (i.p.) of either saline or sActIIBR MSTN antagonist ( $5 \mu \mathrm{g} / \mathrm{g}$ body weight) three times a week (see electronic supplementary material [ESM] for details of purification). Body weight and energy intake (energy intake is equal to food consumption multiplied by metabolisable energy content) were measured twice a week in all trial mice. Mice were killed 1 week after GTT and insulin tolerance test (ITT) analysis, and were not fasted prior to death.

RNA extraction, quantitative real-time $P C R(q P C R)$ and Western blotting RNA extraction, PCR and Western blotting were performed as previously described [28] (see ESM for details).

Insulin and glucose tolerance testing The ITT and GTT were performed as previously described in [15] and [29] respectively (see ESM for details).

Glucose transport in muscle M. extensor digitorum longus (EDL) were rapidly removed from killed mice and were pre-incubated in Krebs-Ringer bicarbonate (KRB) buffer containing $2 \mathrm{mmol} / \mathrm{l}$ pyruvate for $20 \mathrm{~min}$, followed by a further incubation in KRB buffer containing 3,129.3 pmol/l insulin for $30 \mathrm{~min}$. The muscles were then incubated in KRB buffer containing the same concentration of insulin, $1 \mathrm{mmol} / \mathrm{l} 2$ deoxy-D- $\left[{ }^{3} \mathrm{H}\right]$ glucose $(55,500 \mathrm{~Bq} / \mathrm{ml})$ and $7 \mathrm{mmol} / 1 \mathrm{D}-\left[{ }^{14} \mathrm{C}\right]$ mannitol $(11,100 \mathrm{~Bq} / \mathrm{ml})$ at $30^{\circ} \mathrm{C}$ for a further $10 \mathrm{~min}$. The level of glucose transport was subsequently measured as previously described $[29,30]$.

Biochemical analysis See ESM for details of the kits and reagents used for total serum insulin, total cholesterol, triacylglycerol and serum NEFA measurement.
AMPK assays A modified protocol from the Animal Models of Diabetic Complications Consortium [31] was used to measure AMPK activity in protein lysates from muscle tissue. Briefly, after muscle homogenisation, lysates containing $1 \mathrm{mg}$ of muscle protein were centrifuged in a TLA 120.1 rotor (Beckman Coulter, Brea, CA, USA) at $37,000 \mathrm{rpm}(48,000 \times \mathrm{g})$ for $30 \mathrm{~min}$ at $4^{\circ} \mathrm{C}$. The supernatant fraction was then transferred to another centrifuge tube on ice and stirred together with $144 \mathrm{mg}$ of ammonium sulphate, per $\mathrm{ml}$ solution, for an incubation period of $30 \mathrm{~min}$. The sample was then centrifuged at $37,000 \mathrm{rpm}$ for $30 \mathrm{~min}$ at $4^{\circ} \mathrm{C}$, the supernatant fraction was discarded, and the pellet resuspended in $100 \mu$ l of homogenisation buffer.

The SAMS peptide (His-Met-Arg-Ser-Ala-Met-Ser-GlyLeu-His-Leu-Val-Lys-Arg-Arg) utilised in this study was synthesised commercially. The reaction was started by incubating the resuspended ammonium sulphate pellet into a mixture of water, SAMS peptide and radioactive ATP (to $100 \mu \mathrm{l}$ of required working assay mix add $2 \mu \mathrm{l}$ of $370,000 \mathrm{~Bq} / \mathrm{ml} \mathrm{P}^{32}-\gamma$ ATP) for $10 \mathrm{~min}$ at $37^{\circ} \mathrm{C}$. The reaction was terminated by pipetting $15 \mu \mathrm{l}$ of incubation mix onto a $15 \times 15 \mathrm{~mm}$ piece of P81 Whatman filter paper, allowing at least $20 \mathrm{~s}$ for the solution to absorb into paper, then dropping into $150 \mathrm{ml}$ of $1 \%$ phosphoric acid. Finally the filter paper was washed six times, $5 \mathrm{~min}$ each, in $150 \mathrm{ml}$ of $1 \%$ phosphoric acid, followed by a final wash in $50 \mathrm{ml}$ acetone. After drying, the filter paper was measured on a scintillation counter (1450 LSC and luminescence counter; PerkinElmer Life Sciences, Waltham, MA, USA).

\section{Statistical analysis}

The data were analysed using two-way ANOVA and robust regression analysis. Not all the data were normally distributed. Unlike the conventional regression model based on ordinary least square (OLS), the robust model estimates with an iterative reweighted least square (IRWLS) algorithm and generates more reliable estimates about the influence of mouse genotype and diet type on the outcome. The proposed robust model has several advantages over the conventional approach in data analysis. First, it analyses the skewed outcome directly without the need to perform back-transformation for interpretation. This is desirable because arithmetic transformation may not necessarily induce normality in the skewed outcome. Second, it does not discard any observations in the process even if they are confirmed outliers. Finally, it does not impose any strict distributional assumption on the outcome like the generalised linear model (GLM), as there is no precise information about the outcome's probabilistic characteristics $[32,33]$. 


\section{Results}

$\mathrm{Mstn}^{-/-}$mice exhibit a lean phenotype Since $\mathrm{Mstn}^{-1-}$ mice have reduced white adipose tissue (WAT) content, we wanted to investigate the effect of a fat rich diet on muscle metabolism in $\mathrm{Mstn}^{-/-}$mice. In order to do this, WT and $\mathrm{Mstn}^{-1-}$ mice were fed either HFD or regular CD for 12 weeks. During the 12 week diet regimen, no significant difference was noted in the energy intake of WT and $\mathrm{Mstn}^{-/-}$mice fed on HFD when compared with their respective $\mathrm{CD}$ fed controls, but it is important to mention that both the CD and HFD fed $\mathrm{Mstn}^{-/-}$mice had increased energy intake over and above that of both CD and HFD WT mice (Fig. 1a). The body weights, muscle weights and fat pad weights from WT and $\mathrm{Mstn}^{-/-}$mice fed on HFD were compared with their respective CD fed controls. With no change in the amount of food intake, WT mice fed on HFD gained significantly more body weight $(\sim 65 \%)$ as compared with WT mice fed on regular CD $(\sim 35 \%)$ during the 12 week period. In contrast, $\mathrm{Mstn}^{-/-}$mice fed HFD did not significantly increase body weight as compared with regular CD fed $\mathrm{Mstn}^{-/}$mice (Fig. 1b). Feeding HFD did not increase muscle weights significantly either in WT or $\mathrm{Mstn}^{-1-}$ mice (Fig. 1c). It is noteworthy to mention that 7-week-old $\mathrm{Mstn}^{-/-}$mice were very lean and had significantly reduced fat mass as compared with WT mice at the beginning of the HFD trial (Fig. 1e). Upon HFD feeding, the weights of three types of WAT samples (epididymal, retroperitoneal and inguinal) increased significantly during the 12 week period in WT mice; while an increase in the weight of WAT samples was also observed in $\mathrm{Msth}^{-/-}$mice during the 12 week period, the increase was not statisti- a

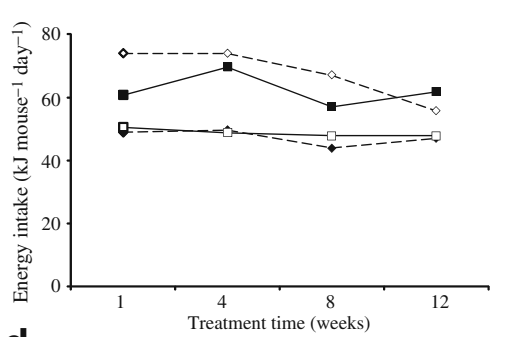

d
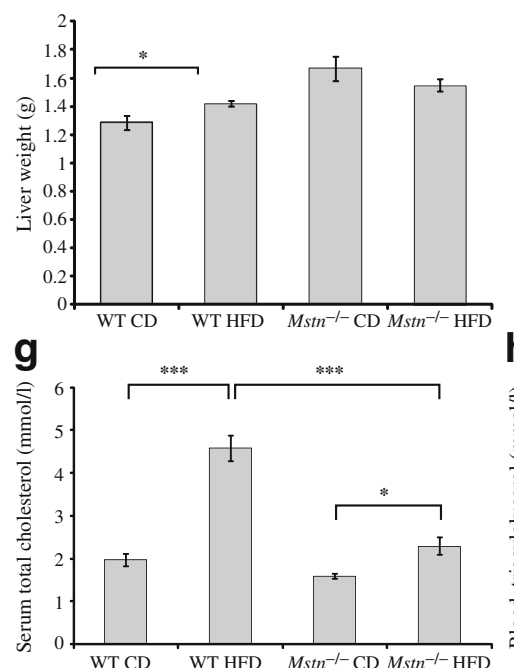
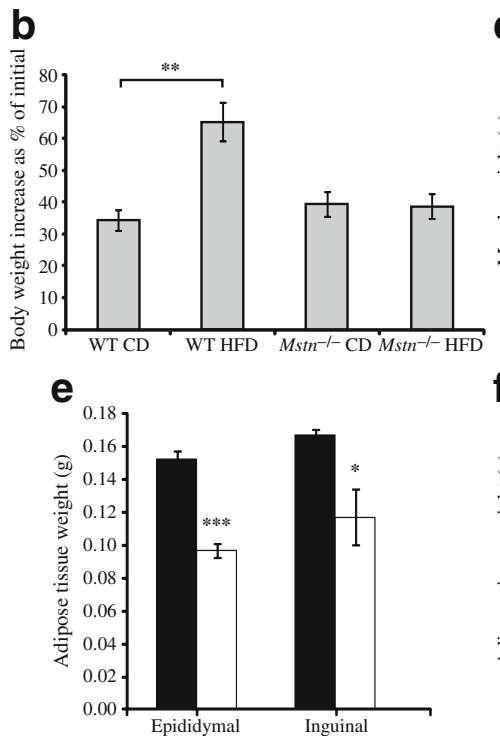

\section{$\mathrm{h}$}

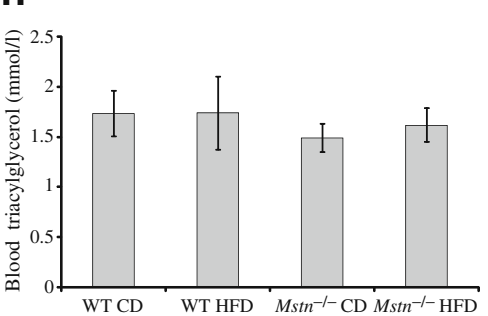

C

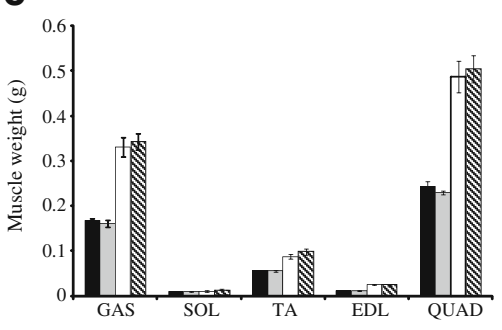

f
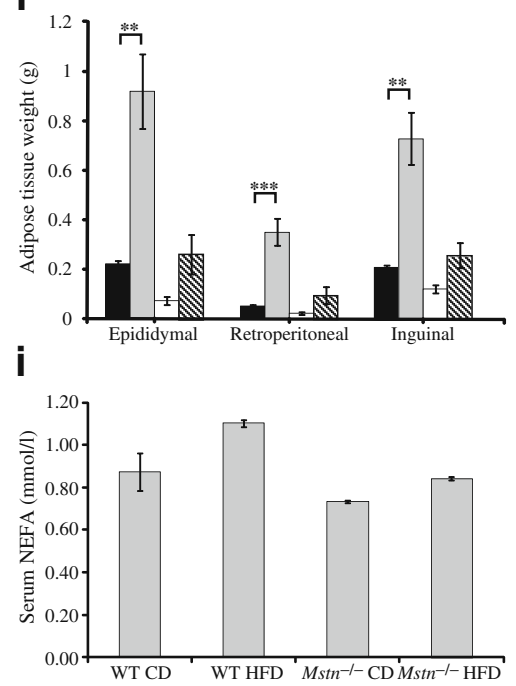

Fig. 1 Lean phenotype in MSTN deficient mice. a Energy intake $\left(\mathrm{kJ}\right.$ mouse ${ }^{-1}$ day $^{-1}$ ) of $\mathrm{Mstn}^{-/-}$mice and WT mice during 12 weeks of $\mathrm{CD}$ and HFD treatment (black diamonds, WT CD; white squares, WT HFD; white diamonds, $\mathrm{Mstn}^{-1-} \mathrm{CD}$; black squares, $\mathrm{Mstn}^{-1-} \mathrm{HFD}$ ). b Body weight increase (expressed as a percentage of initial body weight) observed in $\mathrm{Mstn}^{-/}$mice and WT littermates after 12 weeks of $\mathrm{CD}$ and HFD treatment. c M. gastrocnemius (GAS), M. soleus (SOL), M. tibialis anterior (TA), M. EDL and M. quadriceps (QUAD) weights after 12 weeks of CD and HFD treatment (black bars, WT CD; grey bars WT HFD; white bars $\mathrm{Mstn}^{-/} \mathrm{CD}$; hatched bars, $\mathrm{Mstn}^{-/}$HFD). d Liver weights after 12 weeks of CD and HFD treatment. e Adipose tissue weights of 7-week-old male $\mathrm{Msth}^{-/-}$ mice and WT littermates (black bars, WT; white bars $\mathrm{Mstn}^{-/-}$). f Fat pad weights of $\mathrm{Mstn}^{-1-}$ mice and WT littermates after 12 weeks of CD and HFD treatment (black bars, WT CD; grey bars WT HFD; white bars $\mathrm{Mstn}^{-/-} \mathrm{CD}$; hatched bars, $\mathrm{Mstn}^{-/-}$HFD). g Serum total cholesterol, $\mathbf{h}$ blood triacylglycerol and $\mathbf{i}$ serum NEFA levels of $\mathrm{Mstn}^{-/}$mice and WT mice after 12 weeks of CD and HFD treatment. ${ }^{*} p<0.05,{ }^{* *} p<0.01,{ }^{* * *} p<0.001$. Error bars represent \pm $\operatorname{SEM}(n=5)$ 
cally significant (Fig. 1f). Similarly to the findings in adipose tissue, a significant increase in liver weight was seen in WT mice fed on HFD; however, in contrast, no such increase in liver weight was observed in the $\mathrm{Msth}^{-/-}$ mice fed on HFD (Fig. 1d).

Decreased circulatory cholesterol levels in $\mathrm{Mstn}^{-1-}$ mice To determine whether the reduced fat pad weight in $\mathrm{Mstn}^{-/-}$mice occurred in conjunction with a decrease in lipid levels, we analysed the circulatory levels of cholesterol, triacylglycerol and NEFA. In WT mice fed HFD, there was a $110 \%$ increase in circulatory cholesterol levels when compared with WT CD fed controls, while in $\mathrm{Mstn}^{-/-}$ mice, we observed an increase of $50 \%$ in serum cholesterol levels upon HFD feeding when compared with $\mathrm{Mstn}^{-1-} \mathrm{CD}$ fed controls (Fig. 1g). Therefore, absence of Mstn appears to result in reduced circulating cholesterol levels during HFD feeding. In contrast to the reduced cholesterol levels, we observed no significant change in triacylglycerol and NEFA levels between WT and $\mathrm{Mstn}^{-/-}$mice fed either CD or HFD (Fig. 1h, i).

Lack of MSTN reduces insulin resistance The lean phenotype of $\mathrm{Mstn}^{-/-}$mice, even when fed an HFD, raised the possibility that insulin sensitivity in $\mathrm{Mstn}^{-/}$mice might be maintained even on an HFD. Therefore, the rate of glucose disposal and insulin sensitivity in the WT and $\mathrm{Mstn}^{-1-}$ mice was measured. $\mathrm{Mstn}^{-1-}$ mice had lower fasting blood glucose levels when compared with WT mice (Fig. 2a). Furthermore, WT mice had significantly elevated levels of fasting plasma glucose following HFD treatment ( 8 weeks), when compared with that observed at the start of the trial ( 0 weeks). However, such an increase in fasting plasma glucose levels was not detected in $\mathrm{Mstn}^{-/-}$mice fed on HFD (Fig. 2a). Furthermore, with glucose tolerance testing, a phenotype reflecting insulin resistance, and therefore significantly higher plasma glucose levels, was observed in WT mice when compared with $\mathrm{Mstn}^{-1-}$ mice fed on HFD (Fig. 2b). In addition, $M s t n^{-1}$ mice also showed increased glucose disposal in response to insulin as compared with WT mice during ITT (Fig. 2f).

Increased glucose uptake in the absence of MSTN To determine whether or not disruption of MSTN has a cell autonomous effect on glucose uptake by muscle, we measured glucose uptake in EDL muscle from both WT and $\mathrm{Mstn}^{-/}$ mice. The results show that even at the basal level
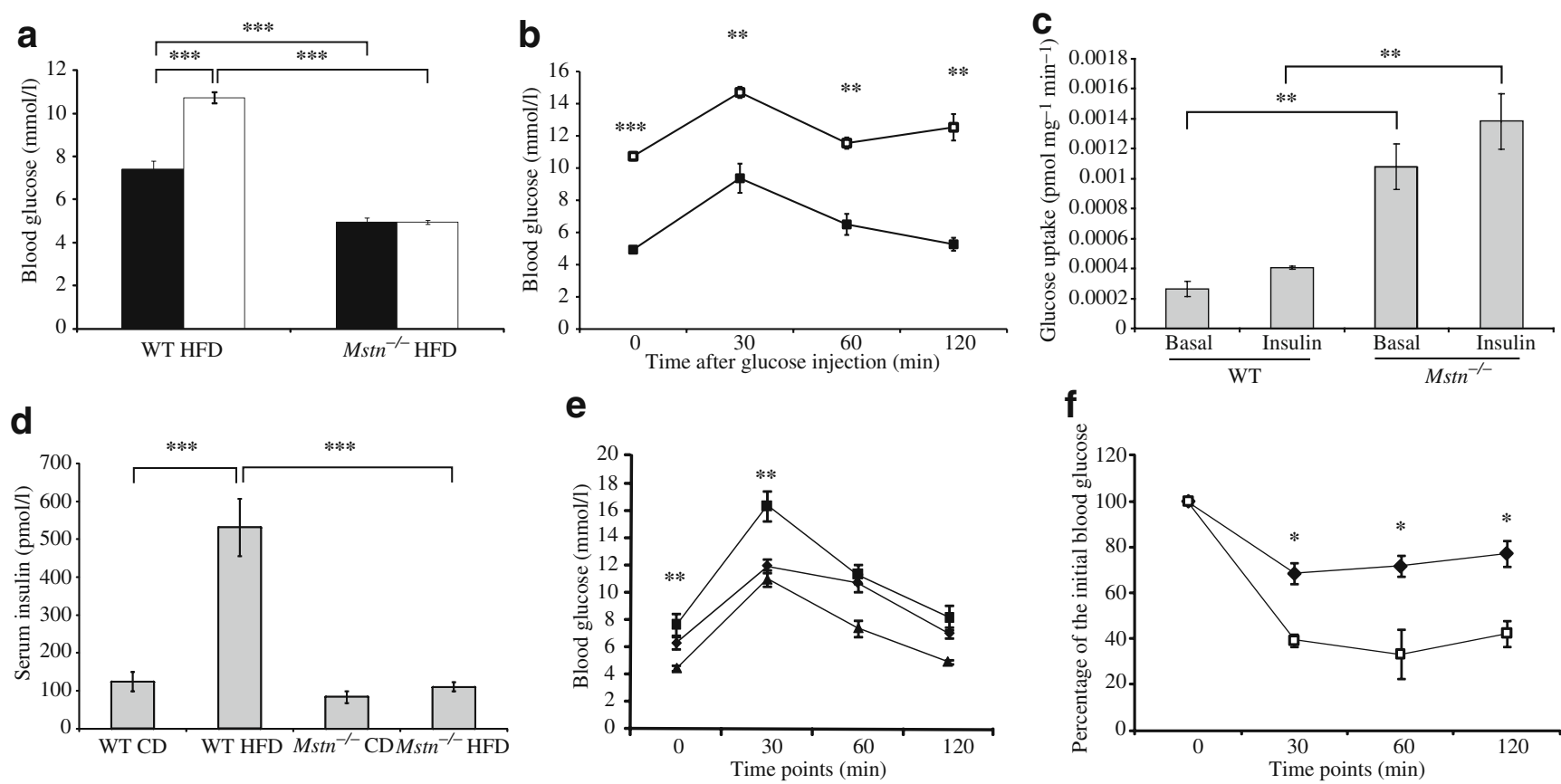

Fig. 2 Lack of MSTN reduces insulin resistance in mice. a Fasting blood glucose levels in WT and $\mathrm{Mstn}^{-1-}$ mice at 0 and 8 weeks of HFD treatment (black bar, 0 weeks; white bar, 8 weeks). b GTT comparing blood glucose levels between WT and Mstn ${ }^{-/}$ mice fed on HFD (white squares, WT HFD; black squares, $\mathrm{Mstn}^{-1-}$ HFD). c Glucose uptake by muscle following insulin stimulation. d Serum insulin levels in WT and $\mathrm{Mstn}^{-1-}$ mice fed on CD or HFD. e Blood glucose levels during GTT on WT mice fed on CD or HFD in the presence of either saline or sActIIBR antagonist (black triangles, WT $\mathrm{CD}+$ saline; black squares, WT HFD+saline; black diamonds, WT HFD+sActIIBR). f Blood glucose levels during an ITT in WT and $\mathrm{Mstn}^{-1-}$ mice. The graph displays blood glucose levels expressed as a percentage of the initial blood glucose level following a $12 \mathrm{~h}$ period of fasting (black diamonds, WT; white squares, $\left.\mathrm{Mstn}^{-1-}\right) .{ }^{*} p<0.05,{ }^{*} p<0.01, * * * p<0.001$. Error bars represent \pm SEM $(n=3)$ 

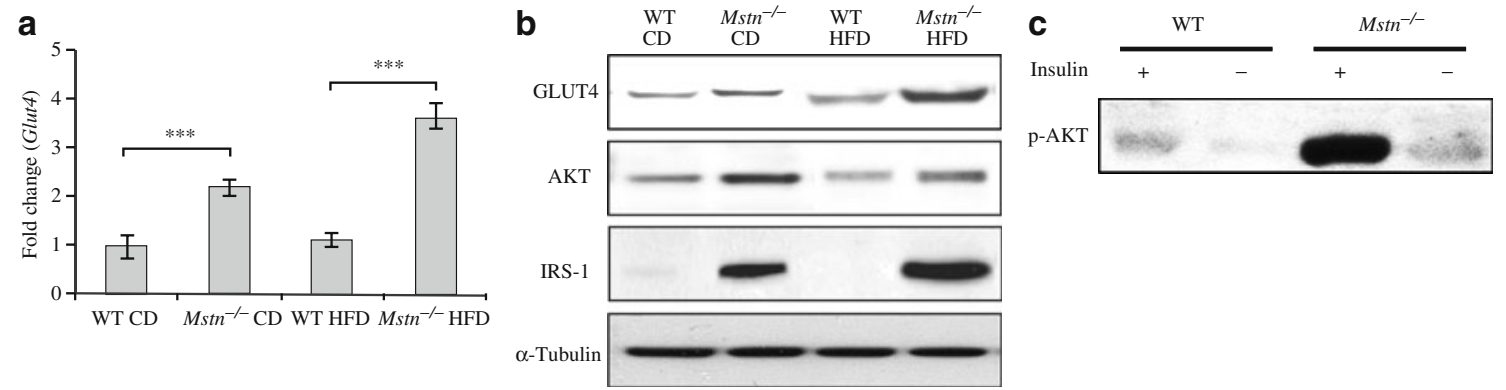

Fig. 3 Molecules involved in the insulin stimulated glucose uptake pathway are upregulated in $\mathrm{Mstn}^{-/-}$mice. a qPCR analysis of the relative mRNA expression of Glut4 in muscle from WT and $\mathrm{Mstn}^{-/}$ mice fed on CD or HFD. b Western blot analysis of GLUT4, AKT and IRS-1 protein levels in BF muscle from WT and $\mathrm{Mstn}^{-/}$mice fed on

(unstimulated by insulin), there is a 2.7 -fold increase in glucose uptake by EDL muscle from $\mathrm{Mstn}^{-/-}$mice compared with WT mice. As expected, insulin was able to stimulate glucose uptake twofold in WT EDL muscle, and only to a minor extent in $\mathrm{Mstn}^{-/}$EDL muscle (Fig. 2c). To determine the molecular basis for the improved glucose uptake in $\mathrm{Mstn}^{-/-}$muscle, we next estimated insulin levels using ELISA. The results show that there was no significant difference in systemic insulin levels between WT and $\mathrm{Msth}^{-/-}$on CD. When challenged with HFD, we observed an increase in circulatory insulin levels in the WT mice;
CD or HFD. c p-AKT protein level in muscle isolated from WT or $\mathrm{Mstn}^{-/-}$mice without (-) or with (+) insulin stimulation. All samples were collected from M. gastrocnemius. ${ }^{* * *} p<0.001$. Error bars represent \pm SEM $(n=3)$

however, in contrast, no significant change in systemic insulin levels was observed in $\mathrm{Mstn}^{-1-}$ mice fed on HFD (Fig. 2d).

Lack of MSTN increases insulin signalling We next performed real-time PCR and Western blot analysis on molecules involved in the insulin-signalling pathway. The results show that there is a significant increase in the expression levels of Glut4 (also known as Slc2a4; Fig. 3a) in muscle from $\mathrm{Mstn}^{-1-}$ mice when compared with WT muscles. When challenged with HFD, we observed further a

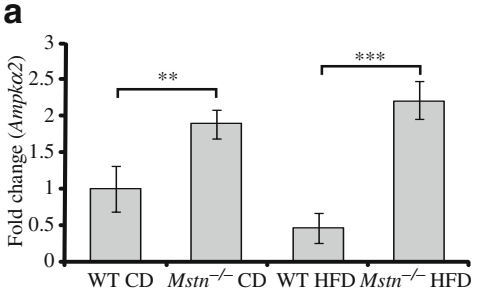

b

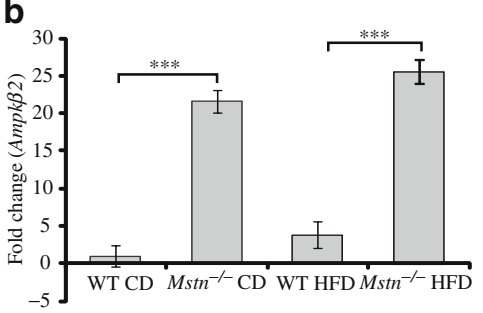

C



d

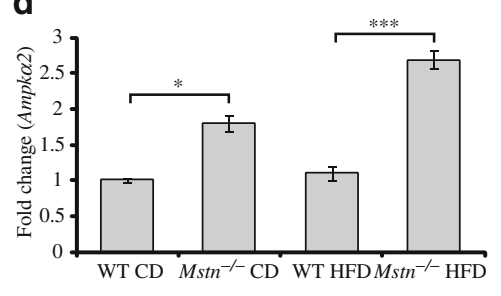

e

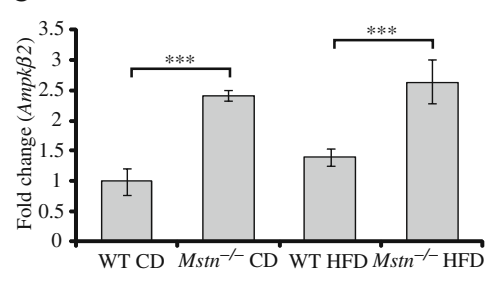

f

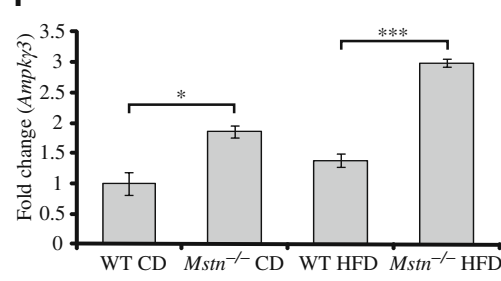

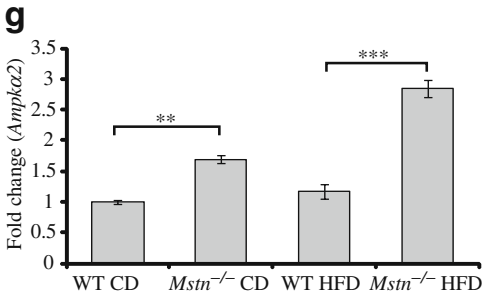

h

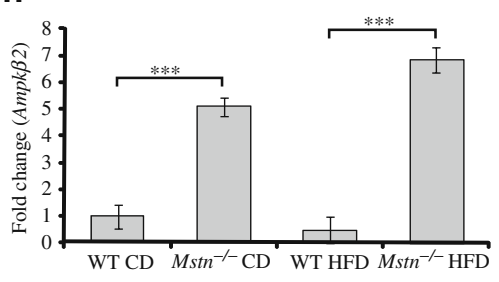

i

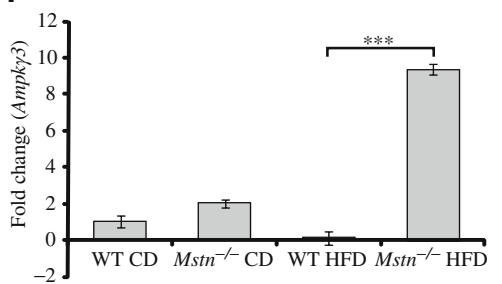

Fig. 4 Increased Ampk mRNA expression is seen in $\mathrm{Mstn}^{-/-}$mice. Relative mRNA expression levels of $A m p k \alpha 2 / \beta 2 / \gamma 3$ in GAS muscle $\mathbf{a}-\mathbf{c}$, WAT (epididymal fat pad) $\mathbf{d}-\mathbf{f}$, and liver $\mathbf{g}-\mathbf{i}$ as measured by
qPCR in WT and $\mathrm{Mstn}^{-/-}$mice fed on CD and HFD. ${ }^{*} p<0.05,{ }^{*} p<$ $0.01, * * * p<0.001$. Error bars represent $\pm \operatorname{SEM}(n=6)$ 
upregulation of Glut4 expression levels in $\mathrm{Mstn}^{-/-}$muscle tissue, whereas no such increase is seen in muscles from the WT mice (Fig. 3a). Western blot analysis further confirmed this result and showed that indeed there was upregulation of GLUT4 protein content in muscle tissue from $\mathrm{Mstn}^{-/-}$fed HFD (Fig. 3b). Western blot analysis also demonstrated an increase in total AKT protein in $\mathrm{Mstn}^{-/-}$muscles (Fig. 3b). As a result, increased p-AKT levels were observed in $\mathrm{Mstn}^{-1-}$ muscles, and when further stimulated with insulin, even greater levels of p-AKT were detected in $\mathrm{Mstn}^{-1-}$ muscles when compared with WT muscles (Fig. 3c). Similarly, we also observed an increase in total IRS-1 protein in $\mathrm{Msth}^{-/-}$muscles (Fig. 3b).

Interfering with MSTN signalling increases tolerance to glucose To ascertain whether or not interfering with MSTN signalling postnatally has a similar effect to that observed in $\mathrm{Mstn}^{-/-}$mice, WT mice fed on HFD were treated with sActIIBR, which has been previously shown to act as a potent antagonist to MSTN activity [34, 35]. Treatment with sActIIBR reduced the insulin resistance seen in mice fed on a HFD. In a GTT test, we observed a significant elevation in blood glucose levels in mice treated with HFD; however, treatment with sActIIBR increased insulin sensitivity and enhanced glucose disposal, despite the fact that the mice were fed HFD. Thus, as can be seen in Fig. 2e, glucose levels were similar between mice fed on regular CD and HFD fed mice treated with sActIIBR (Fig. 2e).

Increased levels of AMPK in Mstn ${ }^{-/}$mice A genome-wide scan for differentially expressed genes revealed that the expression of Ampk was upregulated by several fold in skeletal muscle tissue isolated from $\mathrm{Mstn}^{-/-}$mice (data not shown). To study this further, we quantified the expression of Ampk ( $\alpha 2, \beta 2$ and $\gamma 3$ isoforms) in peripheral tissues from WT and $\mathrm{Mstn}^{-/-}$mice that had been fed CD or HFD. Subsequent qPCR analysis revealed that mRNA expression of all three isoforms of Ampk was significantly upregulated in $\mathrm{Msth}^{-/-}$muscles, liver and adipose tissues (Fig. 4). While HFD feeding further upregulated the mRNA expression of both the catalytic $(\alpha)$ and regulatory $(\beta$ and $\gamma$ ) subunits of Ampk in peripheral tissues of $\mathrm{Mstn}^{-1-}$ mice, no such increase was observed in the peripheral tissues of WT mice fed an HFD (Fig. 4). Western blot analysis further confirmed that there was increased protein production of AMPK $\gamma 3$ in skeletal muscle from $\mathrm{Mstn}^{-/-}$mice (Fig. 5a). Furthermore, there was an increase in the phosphorylation of the $\alpha$-catalytic subunit (AMPK $\alpha)$ in skeletal muscle, liver and adipose tissue from $\mathrm{Mstn}^{-1-}$ mice (Fig. 5b). It was also observed that there was a significant increase in the levels of the AMPK target protein, p-ACC in $\mathrm{Mstn}^{-1-}$ skeletal muscle (Fig. 5a). Consistent with these results, a significant increase in AMPK activity is also detected in
$\mathrm{Mstn}^{-1-}$ skeletal muscle (Fig. 5c). Recently it has been shown that AMPK functions by regulating the expression of genes involved in energy metabolism by acting in coordination with another metabolic sensor, the NADdependent type III deacetylase, SIRT1 [36]. Subsequent real-time PCR analysis indicates that in $\mathrm{Mstn}^{-1-}$ muscles there is increased expression of Sirt1 mRNA (Fig. 5d) and its downstream target gene Pgc-1 $\alpha$ (also known as Ppargcla; Fig. 5e). Consistent with this result, we also observed an increase in SIRT1 and PGC- $1 \alpha$ protein abundance in $\mathrm{Msth}^{--}$muscle collected from mice fed on both CD and HFD when compared with respective WT controls (Fig. 5f, g). Since increased SIRT1 results in deacetylation of PGC- $1 \alpha$, we observed lower levels of acetylated PGC$1 \alpha$ in $\mathrm{Mstn}^{-1-}$ mice fed on both CD and HFD when compared with respective WT controls. In contrast, there was an increase in acetylation of PGC- $1 \alpha$ in WT mice fed HFD when compared with WT CD fed controls (Fig. 5h).

\section{Discussion}

The data presented here suggest a mechanism whereby MSTN negatively regulates the levels of AMPK in peripheral tissues, and thus influences insulin sensitivity (Fig. 6). Increased abundance and activity of AMPK, as observed in $\mathrm{Mstn}^{-1-}$ mice, will lead to enhanced fatty acid oxidation and decreased lipid storage, which is consistent with both the reduced fat accumulation and reduced insulin resistance observed in $\mathrm{Mstn}^{-/-}$mice. These findings reveal MSTN as a novel regulator of fat and glucose metabolism, and suggest that MSTN antagonists may be of benefit in treating obesity and insulin resistance.

In contrast to WT mice, $\mathrm{Mstn}^{-/-}$mice had reduced adipose tissue and failed to accumulate fat in adipose tissue even on an HFD (Fig. 1f). Prolonged high caloric intake leads to metabolic overload and increased triacylglycerol levels, resulting in adipocyte hypertrophy and increased inflammatory response [37]. Macrophage accumulation in turn leads to secretion of pro-inflammatory cytokines such as TNF- $\alpha$, resulting in further requirement of macrophages and impaired triacylglycerol deposition [37]. It is noteworthy to mention that in $\mathrm{Mstn}^{-/-}$mice there are reduced levels of circulating TNF- $\alpha$ [38], raising the possibility that this may be partly responsible for the reduced adipose tissue observed in the absence of Mstn during HFD feeding (Fig. 1f).

Absence of Mstn results in increased Ampk mRNA expression in skeletal muscle, adipose tissue and liver (Fig. 4). Furthermore, we observed increased protein levels of the AMPK $\gamma$ subunit in skeletal muscle, and increased phosphorylation of the catalytic AMPK $\alpha$ subunit in skeletal muscle, WAT and liver from $\mathrm{Mstn}^{-/-}$mice (Fig. 5a, b). 
Consistent with enhanced AMPK production we also observed enhanced activity of AMPK in skeletal muscle (Fig. 5c). AMPK plays a critical role in energy metabolism. Increased AMPK activation leads to phosphorylation and thus inhibition of ACC enzyme activity (Fig. 5a). Reduced ACC enzyme activity leads to reduced malonyl-CoA expression, which further limits fatty acid synthesis and increases fatty acid oxidation. In addition to ACC, it has recently been demonstrated that AMPK controls the expression of genes involved in energy metabolism by modulating another metabolic sensor, the $\mathrm{NAD}^{+}$-dependent type III deacetylase, SIRT1 [36]. AMPK enhances SIRT1 activity by increasing $\mathrm{NAD}^{+}$levels resulting in deacetylation and further activation of SIRT1 targets including PGC$1 \alpha$, leading to increased expression of mitochondrial and lipid metabolism genes [36]. Thus, it is quite possible that the acute actions of AMPK in lipid metabolism in $\mathrm{Mstn}^{-/}$ mice could also be signalled via the decreased acetylation of PGC- $1 \alpha$ observed in $\mathrm{Mstn}^{-/-}$mice (Fig. 5h). However, there is an anomaly; as others and we have shown [39-41], a

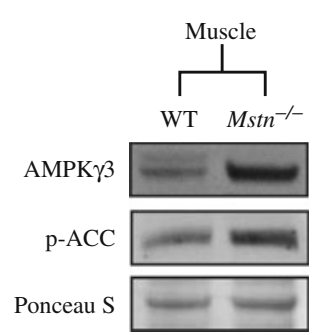

d

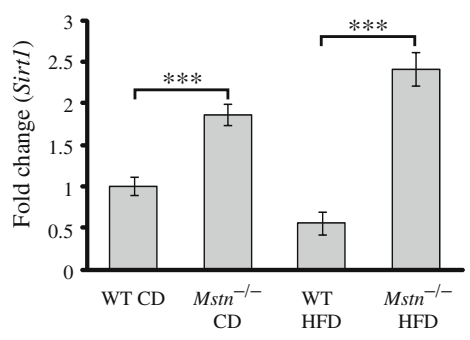

b

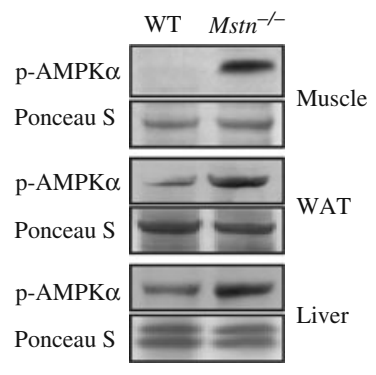

C

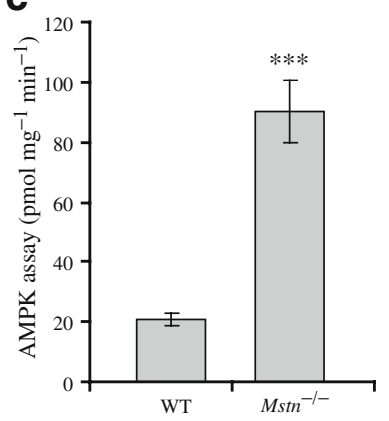

f
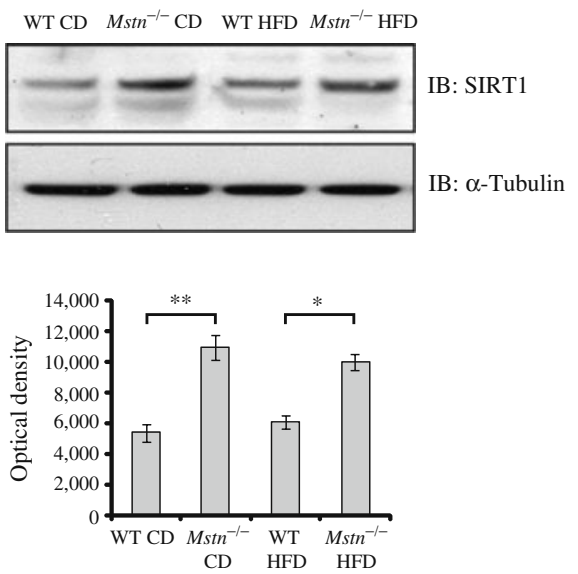

g

$\mathbf{e}$



h
WT CD $M_{s t n^{-/}}$CD WT HFD $M s t n^{-/-}$HFD
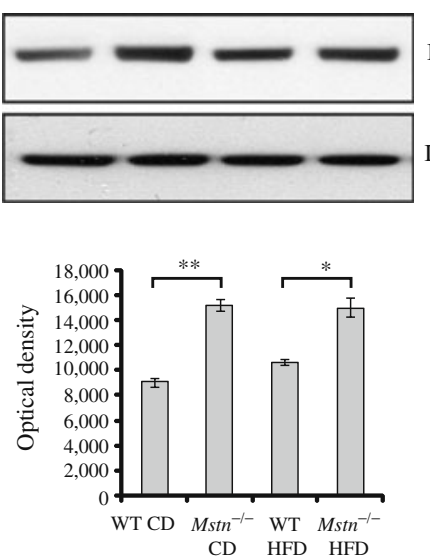

WT CD $\quad \mathrm{Mstn}^{-/-} \mathrm{CD} \quad$ WT HFD $\quad \mathrm{Mstn}^{-/-} \mathrm{HFD}$
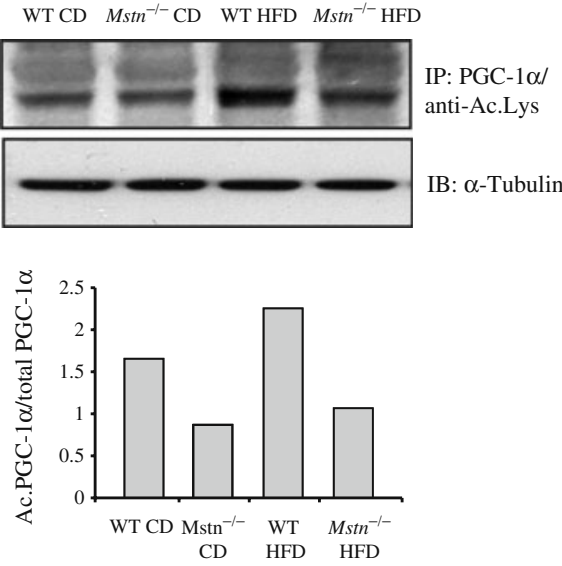

Fig. 5 Increased AMPK activity and augmented SIRT1 signalling is observed in $\mathrm{Mstn}^{-1-}$ mice. a AMPK $\gamma 3$ and p-ACC protein level in muscle of WT and $\mathrm{Mstn}^{-/-}$mice as measured by Western blot. b $\mathrm{p}$-AMPK $\alpha$ protein level in muscle, WAT and liver of WT and $\mathrm{Mstn}^{-1}$ mice as measured by Western blot. c AMPK activity in skeletal muscle as measured by AMPK assay. Relative mRNA expression levels of Sirt $1 \mathbf{d}$ and $P g c-1 \alpha$ e in muscle from WT and $\mathrm{Mstn}^{-/}$mice fed on CD and HFD as measured by qPCR. Western blot analysis of SIRT1 f, PGC-1 $\alpha \mathbf{g}$ and acetyl lysine (Ac.Lys) h protein level in muscle isolated from WT and $\mathrm{Mstn}^{-/-}$mice fed on $\mathrm{CD}$ or HFD. Graphs for SIRT1 and PGC- $1 \alpha$ represent densitometric analysis of each respective Western blot normalised to tubulin. The graph for Ac.Lys represents the level of acetylated PGC- $1 \alpha$ expressed as a proportion of total PGC- $1 \alpha$. All samples were collected from M. gastrocnemius. ${ }^{*} p<0.05,{ }^{* *} p<0.01,{ }^{* * *} p<0.001$. Error bars represent \pm SEM $(n=3)$ 


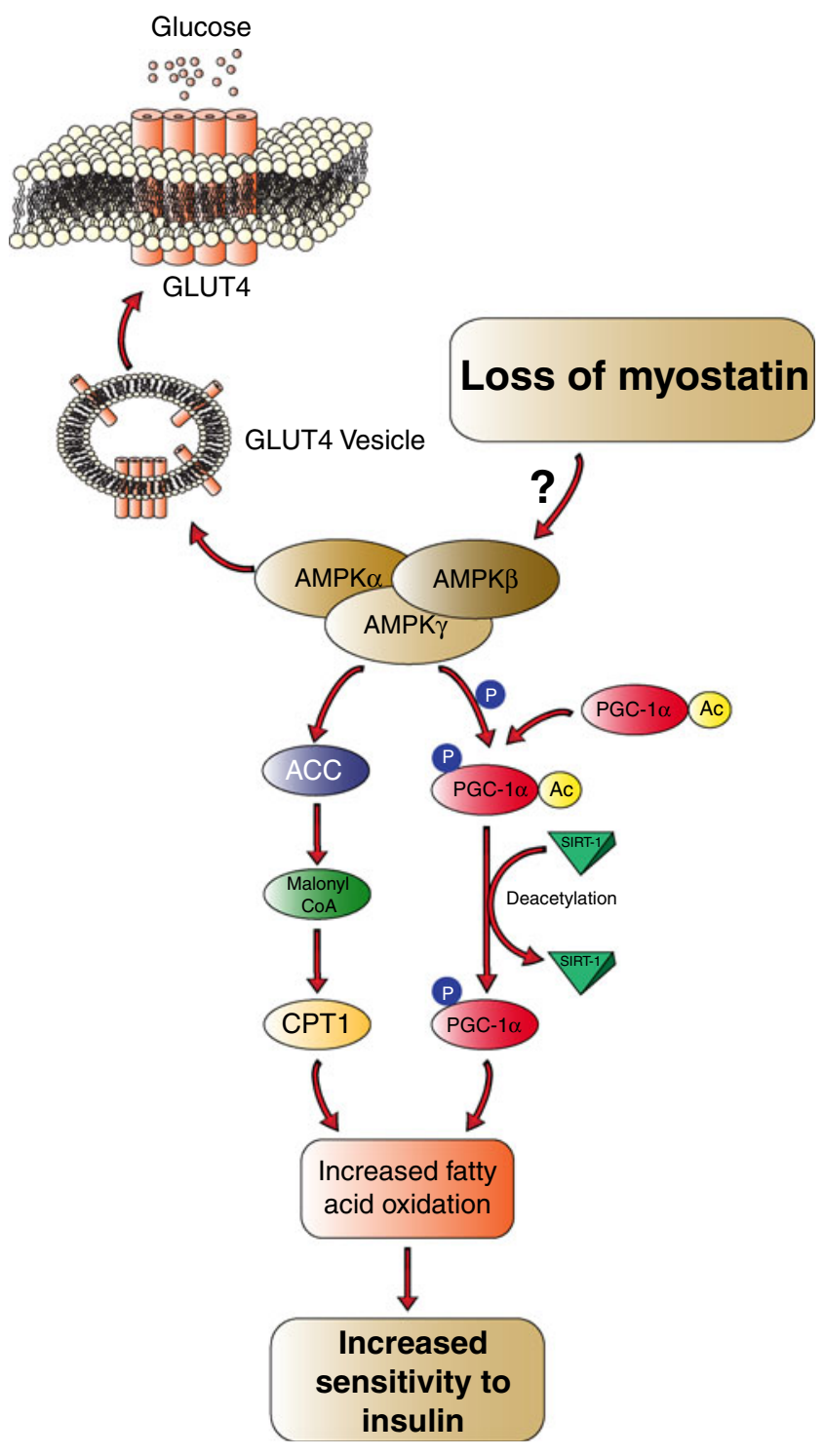

Fig. 6 Proposed mechanism. Loss of MSTN will lead to the activation of AMPK, resulting in the phosphorylation of ACC, leading to reduced malonyl-CoA and increased long chain fatty acyl CoA flux into mitochondria via carnitine palmitoyl transferase-1 (CPT-1) resulting in increased fatty acid oxidation. Activation of AMPK will also phosphorylate PGC- $1 \alpha$, which is further deacetylated by SIRT1, resulting in increased fatty acid oxidation. Activation of AMPK not only leads to increased fatty acid oxidation, it also promotes glucose uptake by accelerating GLUT4 trafficking to the membrane. Therefore, a combination of increased fatty acid oxidation and enhanced glucose uptake will result in enhanced insulin sensitivity in the absence of Mstn

in $\mathrm{Mstn}^{-1-}$ mice there are a greater proportion of fast twitch glycolytic muscle fibres (type IIB). Therefore, one would expect muscles from $\mathrm{Mstn}^{-/-}$mice to be more glycolytic rather than oxidative in nature, as is described here. Interestingly, although predominately fast glycolytic in nature, unpublished data from our lab suggests that there is increased mitochondrial biogenesis, due to fission, in
$\mathrm{Mstn}^{-/-}$fast twitch fibres from the EDL muscle (S. Lokireddy, C. McFarlane, M. Sharma and R. Kambadur, unpublished data). Furthermore, $\mathrm{Mstn}^{-/-}$muscles on average have higher numbers of muscle fibres. Therefore, we speculate that a combination of increased number of mitochondria due to augmented biogenesis and increased muscle fibre number due to hyperplasia are the main driving forces behind the fast glycolytic fibres of $\mathrm{Msth}^{-/-}$ muscles adopting a more oxidative nature, in at least as far as ATP generation is concerned. The benefits of increased fast glycolytic muscle fibre content to metabolic syndrome are clear. Specifically, Izumiya et al. have previously demonstrated that overexpression of a constitutively active form of AKT1 in muscle results in dramatic hypertrophy of type IIB fast glycolytic muscle fibres. Moreover, the increased type IIB muscle mass resulted in resistance to diet-induced obesity, improved sensitivity to insulin and enhanced hepatic fatty acid oxidation [42].

In addition to the reduced adiposity, $\mathrm{Mstn}^{-/-}$mice and, for that matter, mice treated with sActIIBR, the MSTN antagonist, have improved tolerance to glucose and increased sensitivity to insulin (Fig. 2). This increased sensitivity to insulin appears to be due to increased insulin signalling rather than increased insulin secretion, since gene expression and Western blot analysis confirms that there are increased GLUT4 levels and increased phosphorylation of AKT (Fig. 3). We propose that increased insulin signalling in $\mathrm{Mstn}^{-1-}$ mice is due to enhanced AMPK levels and activity. Acute activation of AMPK has been previously shown to result in increased insulin-mediated glucose uptake by increasing GLUT4 levels. Also, prior incubation of epitrochlearis muscles with the AMPK agonist 5aminoimidazole-4-carboxamide ribonucleoside (AICAR) results in a two-fold increase in insulin stimulated glucose transport [25]. Furthermore, AICAR treatment of Zucker rats for 8 weeks improved insulin sensitivity [43]. Moreover, it has been recently shown that ablation of AMPK activity in skeletal muscle exacerbates insulin resistance induced by HFD feeding [30]. Therefore, these prior studies support the hypothesis that enhanced AMPK activity leads to increased insulin signalling in $\mathrm{Mstn}^{-/-}$mice.

In contrast to our results, Chen et al. recently showed that MSTN treatment increases AMPK abundance in vitro [44]. The inconsistencies between our results and the observations made by Chen et al. could be due to the model system used. The addition of MSTN protein (Chen et al.) could result in a negative energy balance via MSTNinduced cachexia [28, 45], leading to a high AMP/ATP ratio resulting in AMPK activation. It is important to highlight that we have utilised an HFD model to study the regulation of glucose metabolism by MSTN and, much like previously published reports $[12,38]$, we find reduced insulin resistance and enhanced glucose uptake, which is 
consistent with the increase in AMPK activity shown in the present manuscript.

In summary, the results presented here demonstrate that lack of MSTN increases insulin signalling and enhances insulin sensitivity in skeletal muscle by increasing AMPK signalling. Furthermore, these results suggest that MSTN antagonists would have utility in resisting obesity and, moreover, in increasing insulin sensitivity during obesity (Fig. 6).

Acknowledgements We would like to thank the Agency for Science, Technology and Research (A*STAR) for funding this project. We would also like to thank J. Swain, Singapore Institute for Clinical Sciences, Singapore, for her comments on the manuscript and R. Choo, Singapore Institute for Clinical Sciences, Singapore, for his invaluable help with statistical analysis of the data presented in this manuscript.

Duality of interest The authors declare that there is no duality of interest associated with this manuscript.

\section{References}

1. McPherron AC, Lawler AM, Lee SJ (1997) Regulation of skeletal muscle mass in mice by a new TGF-beta superfamily member. Nature 387:83-90

2. Sharma M, Kambadur R, Matthews KG et al (1999) Myostatin, a transforming growth factor-beta superfamily member, is expressed in heart muscle and is upregulated in cardiomyocytes after infarct. J Cell Physiol 180:1-9

3. Kambadur R, Sharma M, Smith TP, Bass JJ (1997) Mutations in myostatin (GDF8) in double-muscled Belgian Blue and Piedmontese cattle. Genome Res 7:910-916

4. McPherron AC, Lee SJ (1997) Double muscling in cattle due to mutations in the myostatin gene. Proc Natl Acad Sci USA 94:12457-12461

5. Clop A, Marcq F, Takeda $\mathrm{H}$ et al (2006) A mutation creating a potential illegitimate microRNA target site in the myostatin gene affects muscularity in sheep. Nat Genet 38:813-818

6. Schuelke M, Wagner KR, Stolz LE et al (2004) Myostatin mutation associated with gross muscle hypertrophy in a child. $\mathrm{N}$ Engl J Med 350:2682-2688

7. Langley B, Thomas M, Bishop A, Sharma M, Gilmour S, Kambadur R (2002) Myostatin inhibits myoblast differentiation by down-regulating MyoD expression. J Biol Chem 277:4983149840

8. Thomas M, Langley B, Berry C et al (2000) Myostatin, a negative regulator of muscle growth, functions by inhibiting myoblast proliferation. J Biol Chem 275:40235-40243

9. McCroskery S, Thomas M, Maxwell L, Sharma M, Kambadur R (2003) Myostatin negatively regulates satellite cell activation and self-renewal. J Cell Biol 162:1135-1147

10. Lee SJ, McPherron AC (2001) Regulation of myostatin activity and muscle growth. Proc Natl Acad Sci USA 98:9306-9311

11. Lee SJ, Reed LA, Davies MV et al (2005) Regulation of muscle growth by multiple ligands signaling through activin type II receptors. Proc Natl Acad Sci USA 102:18117-18122

12. Guo T, Jou W, Chanturiya T, Portas J, Gavrilova O, McPherron AC (2009) Myostatin inhibition in muscle, but not adipose tissue, decreases fat mass and improves insulin sensitivity. PLoS ONE 4: e4937
13. Lin J, Arnold HB, Della-Fera MA, Azain MJ, Hartzell DL, Baile CA (2002) Myostatin knockout in mice increases myogenesis and decreases adipogenesis. Biochem Biophys Res Commun 291:701-706

14. McPherron AC, Lee SJ (2002) Suppression of body fat accumulation in myostatin-deficient mice. $\mathrm{J}$ Clin Invest 109:595-601

15. Zhao B, Wall RJ, Yang J (2005) Transgenic expression of myostatin propeptide prevents diet-induced obesity and insulin resistance. Biochem Biophys Res Commun 337:248-255

16. Hittel DS, Axelson M, Sarna N, Shearer J, Huffman KM, Kraus WE (2010) Myostatin decreases with aerobic exercise and associates with insulin resistance. Med Sci Sports Exerc 42:2023-2029

17. Hittel DS, Berggren JR, Shearer J, Boyle K, Houmard JA (2009) Increased secretion and expression of myostatin in skeletal muscle from extremely obese women. Diabetes 58:30-38

18. Bernardo BL, Wachtmann TS, Cosgrove PG et al (2010) Postnatal PPARdelta activation and myostatin inhibition exert distinct yet complimentary effects on the metabolic profile of obese insulinresistant mice. PLoS One 5:e11307

19. Hawley SA, Boudeau J, Reid JL et al (2003) Complexes between the LKB1 tumor suppressor, STRAD alpha/beta and MO25 alpha/ beta are upstream kinases in the AMP-activated protein kinase cascade. J Biol 2:28

20. Ruderman NB, Saha AK, Vavvas D, Witters LA (1999) MalonylCoA, fuel sensing, and insulin resistance. Am J Physiol 276:E1-E18

21. Steinberg GR, Macaulay SL, Febbraio MA, Kemp BE (2006) AMP-activated protein kinase - the fat controller of the energy railroad. Can J Physiol Pharmacol 84:655-665

22. Davies SP, Helps NR, Cohen PT, Hardie DG (1995) 5'-AMP inhibits dephosphorylation, as well as promoting phosphorylation, of the AMP-activated protein kinase. Studies using bacterially expressed human protein phosphatase- $2 \mathrm{C}$ alpha and native bovine protein phosphatase-2AC. FEBS Lett 377:421-425

23. Martin TL, Alquier T, Asakura K, Furukawa N, Preitner F, Kahn BB (2006) Diet-induced obesity alters AMP kinase activity in hypothalamus and skeletal muscle. J Biol Chem 281:1893318941

24. Fujii N, Jessen N, Goodyear LJ (2006) AMP-activated protein kinase and the regulation of glucose transport. Am J Physiol Endocrinol Metab 291:E867-E877

25. Fisher JS, Gao J, Han DH, Holloszy JO, Nolte LA (2002) Activation of AMP kinase enhances sensitivity of muscle glucose transport to insulin. Am J Physiol Endocrinol Metab 282:E18E23

26. Iglesias MA, Ye JM, Frangioudakis G et al (2002) AICAR administration causes an apparent enhancement of muscle and liver insulin action in insulin-resistant high-fat-fed rats. Diabetes 51:2886-2894

27. Jessen N, Pold R, Buhl ES, Jensen LS, Schmitz O, Lund S (2003) Effects of AICAR and exercise on insulin-stimulated glucose uptake, signaling, and GLUT-4 content in rat muscles. J Appl Physiol 94:1373-1379

28. McFarlane C, Plummer E, Thomas M et al (2006) Myostatin induces cachexia by activating the ubiquitin proteolytic system through an NF-kappaB-independent, FoxO1-dependent mechanism. J Cell Physiol 209:501-514

29. Fujii N, Hirshman MF, Kane EM et al (2005) AMP-activated protein kinase alpha2 activity is not essential for contraction- and hyperosmolarity-induced glucose transport in skeletal muscle. J Biol Chem 280:39033-39041

30. Fujii N, Ho RC, Manabe Y et al (2008) Ablation of AMPactivated protein kinase alpha2 activity exacerbates insulin resistance induced by high-fat feeding of mice. Diabetes 57:2958-2966 
31. Consortium AMoDC (2007) Cardiac AMPK assay protocol. www.amdcc.org/shared/showProtocol.aspx?id=56. Accessed 20 January 2010

32. Hamilton LC (1992) How robust is robust regression? Stata Tech Bull 1:21-26

33. Rousseeuw PJ, Leroy AM (1987) Robust regression and outlier detection. Wiley, New York

34. Cadena SM, Tomkinson KN, Monnell TE et al (2010) Administration of a soluble activin type IIB receptor promotes skeletal muscle growth independent of fiber type. J Appl Physiol 109:635642

35. Morrison BM, Lachey JL, Warsing LC et al (2009) A soluble activin type IIB receptor improves function in a mouse model of amyotrophic lateral sclerosis. Exp Neurol 217:258-268

36. Canto C, Gerhart-Hines Z, Feige JN et al (2009) AMPK regulates energy expenditure by modulating $\mathrm{NAD}^{+}$metabolism and SIRT1 activity. Nature 458:1056-1060

37. Xu H, Barnes GT, Yang Q et al (2003) Chronic inflammation in fat plays a crucial role in the development of obesity-related insulin resistance. J Clin Invest 112:1821-1830

38. Wilkes JJ, Lloyd DJ, Gekakis N (2009) Loss-of-function mutation in myostatin reduces tumor necrosis factor alpha production and protects liver against obesity-induced insulin resistance. Diabetes 58:1133-1143
39. Girgenrath S, Song K, Whittemore LA (2005) Loss of myostatin expression alters fiber-type distribution and expression of myosin heavy chain isoforms in slow- and fast-type skeletal muscle. Muscle Nerve 31:34-40

40. Hennebry A, Berry C, Siriett V et al (2009) Myostatin regulates fiber-type composition of skeletal muscle by regulating MEF2 and MyoD gene expression. Am J Physiol Cell Physiol 296:C525C534

41. Siriett V, Platt L, Salerno MS et al (2006) Prolonged absence of myostatin reduces sarcopenia. J Cell Physiol 209:866873

42. Izumiya $\mathrm{Y}$, Hopkins $\mathrm{T}$, Morris $\mathrm{C}$ et al (2008) Fast/glycolytic muscle fiber growth reduces fat mass and improves metabolic parameters in obese mice. Cell Metab 7:159-172

43. Pold R, Jensen LS, Jessen N et al (2005) Long-term AICAR administration and exercise prevents diabetes in ZDF rats. Diabetes 54:928-934

44. Chen Y, Ye J, Cao L, Zhang Y, Xia W, Zhu D (2010) Myostatin regulates glucose metabolism via the AMP-activated protein kinase pathway in skeletal muscle cells. Int J Biochem Cell Biol 42:2072-2081

45. Zimmers TA, Davies MV, Koniaris LG et al (2002) Induction of cachexia in mice by systemically administered myostatin. Science 296:1486-1488 